\title{
Mechanical feedback controls the emergence of dynamical memory in growing tissue monolayers
}

\author{
Sumit Sinha ${ }^{1}$, Xin $\mathrm{Li}^{2}$, Rajsekhar $\mathrm{Das}^{2}$ and D. Thirumalai ${ }^{2}$ \\ ${ }^{1)}$ Department of Physics, University of Texas at Austin, Austin, TX 78712, \\ USA. \\ ${ }^{2)}$ Department of Chemistry, University of Texas at Austin, Austin, TX 78712, \\ $U S A$.
}

The growth of a tissue, which depends on cell-cell interactions and biologically relevant process such as cell division and apoptosis, is regulated by a mechanical feedback mechanism. We account for these effects in a minimal two-dimensional model in order to investigate the consequences of mechanical feedback, which is controlled by a critical pressure, $p_{c}$. A cell can only grow and divide if the pressure it experiences, due to interaction with its neighbors, is less than $p_{c}$. Because temperature is an irrelevant variable in the model, the cell dynamics is driven by self-generated active forces (SGAFs) that are created by cell division. It is shown that even in the absence of intercellular interactions, cells undergo diffusive behavior. The SGAFdriven diffusion is indistinguishable from the well-known dynamics of a free Brownian particle at a fixed finite temperature. When intercellular interactions are taken into account, we find persistent temporal correlations in the force-force autocorrelation function $(F A F)$ that extends over timescale of several cell division times. The time-dependence of the $F A F$ reveals memory effects, which increases as $p_{c}$ increases. The observed non-Markovian effects emerge due to the interplay of cell division and mechanical feedback, and is inherently a non-equilibrium phenomenon.

\section{INTRODUCTION}

Life around us, spanning a bewildering array of length and time scales, is sustained through multicellular processes that are driven by non-equilibrium events such as cell growth and cell division 12. Although known for a long time ${ }^{3}$, several recent experimental studies have emphasized that growth and division in cell collectives are governed by local stresses that the cells experience ${ }^{4 / 7}$. A manifestation of coupling of growth and division to local stress is the deviation of growth law of the cell collective from exponential law ${ }^{6}$. These experiments suggest that there must exist mechanical feedback between the local stress and cell division.

In a series of papers, we showed that single cells in a collective exhibit anomalous dynamics due to local stressdependent cell growth and division 14 In the present study, we explore the dependence of mechanical feedback, mediated by stress threshold $p_{c}$, on the dynamics of single cells. A recent interesting study $\frac{15}{15}$, has shown that mechanical feedback regulates the physical properties of jammed cell collectives, which supports experimental findings 4 . However, how the mechanical feedback regulates individual cell migration in a collective is unknown, and is the problem which we address in this study.

Using a two-dimensional off-lattice agent-based simulation model, we explore the role of mechanical feedback $\left(p_{c}\right)$ on single-cell dynamics. The central results of the present study are: (i) In the absence of cell growth and division, the dynamics is solely governed by short-ranged two body interactions. In this limit, the cells in the longtime behave like a glass-like solid. (ii) In the presence of cell division, with systematic interactions absent, the cells exhibit Markov dynamics resulting in diffusive motion at long times. This finding is surprising because there is no thermal motion (temperature is an irrelevant variable). This is different from a free Brownian particle where temperature randomizes the particle motion. (iii) When both cell division and systematic interactions control the collective movement in tandem, the dynamics is regulated by the mechanical feedback that is parameterized using $p_{c}$. To quantify the dynamics, we calculated the force auto-correlation function (FAF) inspired by works in the theory of chemical reactions in liquids $\frac{16}{18}$. We show FAF increases when $p_{c}$ is increased. The emergence of long time correlation in the FAF shows departure from Markov dynamics, and is suggestive of memory effects in growing cell collectives. (iv) The persistence of trajectories of individual cells increases as $p_{c}$ is increased. The trajectories are strikingly different from simple Brownian motion. The enhanced persistence in cell dynamics, as $p_{c}$ increases, is the origin of memory in active systems. Taken together, the present study establishes how mechanical feedback coupled with cell growth and division leads to non-Markovian cell dynamics whose importance has not been appreciated before.

\section{METHODS}

We simulated the spatial and temporal dynamics of a two dimensional (2D) growing tissue using agent-based off-lattice model in which the cells are represented as interacting deformable disks. This simplified assumption of representing cells as deformable disks was also used in previous studies ${ }^{19}$, although the details differ. In the model, the cells grow stochastically in time and divide upon reaching a critical size $\left(R_{m}\right)$, the mitotic radius. The cell-to-cell interaction is characterized by elastic and adhesive forces. We also consider cell-to-substrate damp- 
ing as a way of accounting for the effects of friction experienced by a moving cell by the substrate.

Physical Interactions : Each cell is modeled as a deformable disk with a time dependent radius. A cell is characterized by physical properties such as the radius, elastic modulus, membrane receptor and ligand concentration. In addition, the cells attract each other through E-Cadherin mediated adhesive interactions. This model is inspired by previous works on 3D off-lattice multicellular tumor growth models 5$]$ 13/20|21. The elastic (repulsive) force between two disks with radii $R_{i}$ and $R_{j}$ is given by,

$$
F_{i j}^{e l}(t)=\frac{h_{i j}^{3 / 2}(t)}{\frac{3}{4}\left(\frac{1-\nu_{i}^{2}}{E_{i}}+\frac{1-\nu_{j}^{2}}{E_{j}}\right) \sqrt{\frac{1}{R_{i}(t)}+\frac{1}{R_{j}(t)}}},
$$

where $E_{i}$ and $\nu_{i}$, respectively, are the elastic modulus and Poisson ratio of cell $i$. The overlap between the disks, if they interpenetrate without deformation, is $h_{i j}$, which is given by $\max \left[0, R_{i}+R_{j}-\left|\vec{r}_{i}-\vec{r}_{j}\right|\right]$ with $\left|\vec{r}_{i}-\vec{r}_{j}\right|$ being the center-to-center distance between the two disks.

Cell adhesion, mediated by receptors on the cell membrane, is the process by which cells can attach to one another. For simplicity, we assume that the receptor and ligand molecules are evenly distributed on the cell surface. Consequently, the magnitude of the adhesive force, $F_{i j}^{a d}$, between two cells $i$ and $j$ is expected to scale as a function of their contact line-segment, $L_{i j}$. Keeping the $3 \mathrm{D}$ model as a guide ${ }^{8}$, we calculate $F_{i j}^{a d}$ using,

$$
F_{i j}^{a d}=L_{i j} f^{a d} \frac{1}{2}\left(c_{i}^{r e c} c_{j}^{l i g}+c_{j}^{r e c} c_{i}^{l i g}\right),
$$

where the $c_{i}^{r e c}\left(c_{i}^{\text {lig }}\right)$ is the receptor (ligand) concentration (assumed to be normalized with respect to the maximum receptor or ligand concentration so that $0 \leq$ $\left.c_{i}^{r e c}, c_{i}^{l i g} \leq 1\right)$. The coupling constant $f^{a d}$ allows us to rescale the adhesion force to account for the variabilities in the maximum densities of the receptor and ligand concentrations. We calculate the contact length, $L_{i j}$, using the length of contact between two intersecting circles, $L_{i j}=\frac{\sqrt{\left(\left|4 r_{i j}^{2} R_{i}^{2}-\left(r_{i j}^{2}-R_{j}^{2}+R_{i}^{2}\right)^{2}\right|\right)}}{r_{i j}}$. Here, $r_{i j}$ is the distance between cells $i$ and $j$. As before, $R_{i}$ and $R_{j}$ denote the radius of cell $i$ and $j$.

Repulsive and adhesive forces considered in Eqs.(1) and (2) act along the unit vector $\mathbf{n}_{i j}$ pointing from the center of cell $j$ to the center of cell $i$. The total force on the $i^{t h}$ cell is given by the sum over its nearest neighbors $(N N(i))$,

$$
\mathbf{F}_{i}=\Sigma_{j \in N N(i)}\left(F_{i j}^{e l}-F_{i j}^{a d}\right) \mathbf{n}_{i j} .
$$

The nearest neighbors satisfy the condition $R_{i}+R_{j}-$ $\left|\mathbf{r}_{i}-\mathbf{r}_{j}\right|>0$. Figure 1 a shows the plot of the total force as a function of inter-cellular distance.

Three Scenarios: In order to elucidate the dramatically different dynamical behavior, we consider three limits. (I) The collective movement arising solely from the systematic forces, given in Eq. 3. (II) Cell movement with $\mathbf{F}_{i}=0$ (no inter-cellular interactions) but allowing for cell division and growth. Note that since intercellular interactions are absent, mechanical feedback $\left(p_{c}\right)$ does not play a role. In this limit, we show that the dynamics can only arise due to active forces generated upon cell division. The limits (I) and (II) are not relevant in describing collective movements in Multicellular Spheroids (MCSs) ${ }^{22}$ or evolving cell monolayers ${ }^{6}$. (III) In this limit, we not only include interactions between cells (Eq. 3) but also allow for cell growth, division and apoptosis. Most importantly, the time-dependent growth of the tissue colony is limited by mechanical feedback, which prohibits the biologically important process of cell growth and division if the local stress on a cell exceeds a critical non-zero value, $p_{c}$.

Equation of Motion: The damped dynamics of the $i^{t h}$ cell is computed based on the equation of motion,

$$
\dot{\mathbf{r}}_{i}=\frac{\mathbf{F}_{i}}{\gamma_{i}}
$$

Here, $\gamma_{i}$ is the friction coefficient of the $i^{\text {th }}$ cell. We assume $\gamma_{i}$ to be equal to $\gamma_{o} R_{i}(t)$, where $\gamma_{o}$ is a constant. This form of $\gamma_{i}$ is inspired from the simulations of three dimensional models for solid tumor where $\gamma_{i}=6 \pi \eta R_{i}$ with $\eta$ being the viscosity. Note, we do not consider the effect of temperature (set to zero in the simulations) as we assume the friction coefficient, that in reality arises from the extracellular matrix in $3 \mathrm{D}$ or substrate in $2 \mathrm{D}$, to be so high ${ }^{19}$ that thermal motion is irrelevant. The equation of motion in Eq. 4 is similar to the case for soft granular materials where the role of temperature is neglected ${ }^{23}$. However, it is crucial to note that in the growth of the tissue colony, scenarios II and III in our case, there is a self-generated active force (SGAF) that arises due to the biologically important processes of cell growth and division 10 .

Cell growth, division and apoptosis: In our model, cells can be either in the dormant $(D)$ or in the growth $(G)$ phase depending on the local pressure associated with a cell (Figure 1(b)). Using Irving-Kirkwood definition, we track the pressure $\left(p_{i}\right)$ experienced by the $i^{\text {th }}$ cell due to contact with its neighbors 24 . The expression for $p_{i}$ is given by,

$$
p_{i}=\frac{1}{2} \Sigma_{j \in N N(i)} \frac{\mathbf{F}_{i j} \cdot \mathbf{d} \mathbf{r}_{i j}}{A_{i}},
$$

where $A_{i}=\pi R_{i}^{2}$, is the area of the cell. If the local pressure, $p_{i}$, exceeds a critical limit $\left(p_{c}\right)$ the cell stops growing and enters the dormant phase. Note, the cell can switch back to a growing phase if $\frac{p_{i}}{p_{c}}<1$ as the tissue evolves. The critical pressure $p_{c}$, serves as a mechanical feedback, which is known to regulate the growth of tissues ${ }^{7}$.

For growing cells $\left(\frac{p_{i}}{p_{c}}<1\right)$, their area increases at a constant rate $r_{A}$. The cell radius is updated from a Gaussian distribution with the mean rate $\dot{R}=(2 \pi R)^{-1} r_{A}$. Over the cell cycle time $\tau$,

$$
r_{A}=\frac{\pi\left(R_{m}\right)^{2}}{2 \tau}
$$


where $R_{m}$ is the mitotic radius. The cell cycle time $(\tau)$ is related to the growth rate $\left(k_{b}\right)$ by $\tau=\frac{\ln 2}{k_{b}}$. A cell divides once it grows to the fixed mitotic radius $\left(R_{m}\right)$. To ensure area conservation, upon cell division, we use $R_{d}=R_{m} 2^{-1 / 2}$ as the radius of the daughter cells. The two resulting cells are placed at a center-to-center distance $d=2 R_{m}\left(1-2^{-1 / 2}\right)$. The direction of the new cell location is chosen randomly from a uniform distribution on the unit circle. One source of stochasticity in the cell movement in our model is due to random choice for the mitotic direction. In our simulations, the cells may undergo apoptosis at the rate $k_{a}$. Throughout this work, the apoptosis rate was fixed to $10^{-6} s^{-1}$. Table I depicts the parameters used in the simulations.

\begin{tabular}{|c|c|c|}
\hline Parameters & Values & References \\
\hline Timestep $(\Delta t)$ & $10 \mathrm{~s}$ & This paper \\
\hline $\begin{array}{l}\text { Critical Radius for Division } \\
\left(R_{m}\right)\end{array}$ & $5 \mu \mathrm{m}$ & 8121 \\
\hline Friction coefficient $\left(\gamma_{o}\right)$ & $0.1 \mathrm{~kg} /(\mu \mathrm{m} \mathrm{s})$ & This paper \\
\hline Cell Cycle Time $(\tau)$ & $54000 \mathrm{~s}$ & \begin{tabular}{|l|l|}
$8 / 25$ & 27 \\
\end{tabular} \\
\hline Adhesive Coefficient $\left(f^{a d}\right)$ & $10^{-4} \mu \mathrm{N} / \mu \mathrm{m}$ & This paper \\
\hline $\begin{array}{l}\text { Mean Cell Elastic Modulus } \\
\left(E_{i}\right)\end{array}$ & $10^{-3} \mathrm{MPa}$ & 828 \\
\hline \begin{tabular}{llll|} 
Mean Cell Poisson Ratio \\
$\left(\nu_{i}\right)$
\end{tabular} & 0.5 & 8121 \\
\hline Apoptosis Rate $\left(k_{a}\right)$ & $10^{-6} \mathrm{~s}^{-1}$ & 8 \\
\hline $\begin{array}{l}\text { Mean Receptor Concentra- } \\
\text { tion }\left(c^{r e c}\right)\end{array}$ & 1.0 & 8 \\
\hline $\begin{array}{l}\text { Mean Ligand Concentration } \\
\left(c^{\text {lig }}\right)\end{array}$ & 1.0 & 8 \\
\hline
\end{tabular}

TABLE I: The parameters used in the simulation.

Initial Conditions: We begin the simulations by placing 100 cells on a 2D plane whose coordinates are chosen from a normal distribution with mean zero and standard deviation $25 \mu \mathrm{m}$. All the parameters apart from critical pressure $\left(p_{c}\right)$ are fixed.

\section{RESULTS}

\section{A. Markov dynamics in the presence of delta-correlated random force}

For comparison, we briefly summarize the well-known result for a stochastic process (over-damped Langevin equation) in one dimension for a free Brownian particle. The equation of motion is,

$$
\frac{d x}{d t}=\sqrt{2 D} \eta(t)
$$

where the random force obeys $\langle\eta(t)\rangle=0$ and $\left\langle\eta(t) \eta\left(t^{\prime}\right)\right\rangle=\delta\left(t-t^{\prime}\right)$. The position of the particle is $x$. The solution $P(x, t)$, for probability density for finding the particle at $x$ at time $t$, is given by

$$
P(x, t)=\frac{1}{\sqrt{4 \pi D t}} e^{-\frac{x^{2}}{4 D t}} .
$$

Here, we have assumed that the particle was at the origin at $t=0, P(x, 0)=\delta(x)$. The moments of $P(x, t)$, which serve as the physical observables in cell tracking experiments ${ }^{22}$, are readily calculated. For instance, the first moment $\langle x\rangle$ is given as

$$
\langle x(t)\rangle=\int_{-\infty}^{\infty} x P(x, t)=0 .
$$

The second moment $\left\langle x^{2}\right\rangle$, also called the mean-squared displacement (MSD), is non-zero and is given as

$$
\left\langle x^{2}(t)\right\rangle=\int_{-\infty}^{\infty} x^{2} P(x, t)=2 D t .
$$

The dynamics of a free Brownian particle is an example of a Markov process. There is no memory because $P(\delta x(t))=\mathcal{N}(0, \sqrt{2 D \delta t})$ is independent of $x(t)$, where $\delta x(t)=x(t+\delta t)-x(t)$ is the displacement of the particle from time $t$ to $t+\delta t$ and $\mathcal{N}(0, \sqrt{2 D \delta t})$ is the normal distribution with zero mean and variance $2 D \delta t$. This example sets the stage for exploring the emergent force auto-correlation $(F A F)$, with long temporal correlation, in an expanding tissue.

\section{B. Role of physical interactions and cell division}

We first investigate the consequences of physical interactions and cell division when they are not coupled to one another.

(a) Physical Interactions (limiting case I): When the interactions between cells are based only on the systematic interactions, as given in Eqs. 1 and 2 without cell division and apoptosis, the dynamics of the interacting cells is governed by the elastic timescale $\frac{\gamma}{E R_{m}}$. In the absence of cell growth, division and apoptosis, the number of cells, $N(t)$, is a constant, which is confirmed in Figure 2a. Figure $2 \mathrm{~b}$ shows the plot of mean-square displacement, $\Delta(t)$, defined as

$$
\Delta(t)=\left\langle\frac{1}{N} \sum_{i=1}^{N}\left(\mathbf{r}_{i}(t)-\mathbf{r}_{i}(0)\right)^{2}\right\rangle,
$$

where $\langle\ldots\rangle$ denotes the ensemble average over 20 simulation runs and $N$ is the initial 100 cells. Figure 2 a shows that $\Delta(t)$ relaxes rapidly to a plateau on a time scale $\approx \frac{\gamma}{E R_{m}}$. Usually, $\Delta(t) \sim t^{\alpha}$, with $\alpha=0$, is indicative of solid-like behavior. Figure $2 \mathrm{~b}$ shows that in the long time limit, $t>>\frac{\gamma}{E R_{m}}, \alpha=0$, and hence the cell collective behaves as a solid in the sense there is absence of diffusion. Note, the dynamics is performed under athermal (temperature is not relevant) open boundary conditions and the scale of systematic interactions are short-ranged $\left(\approx R_{m}\right)$. Hence, the cells cannot move after the initial relaxation process.

(b) Effect of cell division (limiting case II): In this limit, during each cell division event, a cell is displaced by the distance $\approx R_{m}$, randomly in space. Hence, 
when the time evolution of cell colony is governed solely by cell division (absence of apoptosis and systematic interactions) we expect that with successive cell divisions a cell would undergo a random walk that is uncorrelated in time and space. In other words, it would behave as a Brownian particle due to the SGAF induced by cell division. This is purely a non-equilibrium dynamical process. As a result, we expect that $\Delta(t)=D_{\text {eff }} t$ at long times. Because in this limiting case $\tau$ is the only time scale and $R_{m}$ is the only length scale, we obtain $D_{\text {eff }}=\frac{R_{m}^{2}}{\tau}$. In the absence of systematic interactions the pressure (Eqn. 5 on an individual cell is zero, all the cells grow and divide independently. Hence, the number of cells, $N(t)$ increases exponentially (see Figure $2 \mathrm{c}$ ). In this limit,

$$
\frac{d N(t)}{d t}=k_{b} N(t)
$$

and therefore $N(t)=N_{o} e^{k_{b} t}$. Interestingly, in accord with the arguments given above, the dynamics of individual cells is diffusive in this limit and the mean squared displacement $\Delta(t)$ is given by

$$
\Delta(t)=\frac{R_{m}^{2}}{\tau} t
$$

as shown in Figure $2 \mathrm{~d}$. Although there is no thermal motion, the scaling behavior of $\Delta(t)$, is similar to the standard Brownian dynamics given by Eqn. 10. The dynamics is diffusive because during every cell division the cell is displaced by distance $R_{m}$ randomly, and hence mimics a Brownian motion in two dimensions. Note, when only cell apoptosis is present $\left(k_{b}=0\right)$, the cells do not move, and $N(t)$ decreases exponentially to zero at the rate $k_{a}$.

The dynamics is non-trivial when all the components (systematic forces, cell division and apoptosis, and mechanical feedback) are included. For this case, the growing tissue develops a core where the cells are jammed, and exhibit glass-like dynamics ${ }^{11}$. In contrast, the cells in the periphery are predominantly in the growth $(G)$ phase. As a result, the cells exhibit anomalous spatially heterogeneous dynamics with super-diffusive (sub-diffusive) periphery (core) $)^{11}$. Our previous work has shown that this dynamical phase separation arises due to SGAFs that arise due to local stress-regulated cell growth and division 10 . In the model, cell division and growth are regulated by the mechanical feedback parameter $p_{c}$, the consequences of which is explored in the following section.

\section{Highly correlated force correlations in an expanding tissue}

For a simple Brownian motion, the force is deltacorrelated and hence the dynamics is Markovian (see Eqn. 7). Therefore, a signature of such dynamics is the fast decay of force auto-correlation function $(F A F)$, in comparison to the smallest time-scale of the problem, which in the present case is $\frac{\gamma}{E R_{m}}$. To explore the nature of the dynamics, when both cell division and systematic interactions are present in tandem, we calculated $F A F$ $\left(t^{*}\right)$ given as

$$
F A F\left(t^{*}\right)=\frac{\left\langle\mathbf{F}\left(t+t^{*}\right) \cdot \mathbf{F}(t)\right\rangle_{t}}{\langle\mathbf{F}(t) \cdot \mathbf{F}(t)\rangle_{t}}
$$

Here, $\mathbf{F}(t)$ is the force on the cell at time $t$ and $\langle\ldots\rangle_{t}$ is the time average, and $t^{*}$ is the delay (or waiting) time. The time averaging is performed over $\approx 2,000$ cells. Figure 3 shows the plot of FAF for $p_{c}=10^{-3} \mathrm{Nm}^{-1}, 10^{-4} \mathrm{Nm}^{-1}$ and $10^{-5} \mathrm{Nm}^{-1}$. Figure 3 shows that the FAF decays on two time scales: long $\left(\sim \frac{1}{k_{b}-k_{a}}\right)$ and short $\left(\frac{\gamma}{E R_{m}}\right)$. In order to extract the two time scales, we fit FAF with $A e^{\frac{-t^{*}}{\tau_{c}}}+B$ in both the regimes.

In the short time regime (see the inset of Figure 3), for $p_{c}=10^{-3} \mathrm{Nm}^{-1}, A=0.5, \tau_{c}=\frac{1.2 \gamma}{E R_{m}}$ and $B=0.41$. For $p_{c}=10^{-4} \mathrm{Nm}^{-1}, A=0.75, \tau_{c}=\frac{0.97 \gamma}{E R_{m}}$ and $B=0.16$. Lastly, for $p_{c}=10^{-5} \mathrm{Nm}^{-1}, A=0.81, \tau_{c}=\frac{0.95 \gamma}{E R_{m}}$ and $B=0.11$. As anticipated, we find that in the short time regime, the relaxation time is approximately close to the elastic time scale $\frac{\gamma}{E R_{m}}$, which is negligible compared to $\frac{1}{k_{b}-k_{a}}$. However, in the long time limit, FAF shows memory effects, especially for $p_{c}=10^{-3} \mathrm{Nm}^{-1}$. For $p_{c}=10^{-3} \mathrm{Nm}^{-1}, A=0.41, \tau_{c}=\frac{2.2}{k_{b}-k_{a}}$ and $C=-0.06$. For $p_{c}=10^{-4}, A=0.12, \tau_{c}=\frac{2.3}{k_{b}-k_{a}}$ and $B=-0.02$. Lastly, for $p_{c}=10^{-5} \mathrm{Nm}^{-1}, A=0.04, \tau_{c}=\frac{0.2}{k_{b}-k_{a}}$ and $B \approx 0$. For $p_{c}=10^{-5} \mathrm{Nm}^{-1}, \mathrm{~A}$ is negligible which indicates the absence of memory. In the other two cases, A for $p_{c}=10^{-3} \mathrm{Nm}^{-1}$ is four times larger than for $p_{c}=10^{-4} \mathrm{Nm}^{-1}$. Larger magnitude of FAF in the long time regime leads to higher degree of migration for $p_{c}=10^{-3} \mathrm{Nm}^{-1}$. The emergence of highly correlated forces elucidates the departure from Markovian dynamics in a system comprising of many interacting cells whose time evolution occurs under non-equilibrium conditions, and absence of fluctuation-dissipation theorem 14 .

\section{Persistence of trajectories increases on increasing $p_{c}$}

To better understand, the significance of memory effects that are embodied in FAF, we probed the trajectories of individual cells in the growing tissue colony. We investigated the three cases: $p_{c}=10^{-5} \mathrm{~N} / \mathrm{m}, 10^{-4} \mathrm{~N} / \mathrm{m}$ and $10^{-3} \mathrm{~N} / \mathrm{m}$ in Figure 4 Note, the trajectories are for the initial 100 cells with the identical initial conditions and the total simulation time. Thus, the differences in the trajectories emerge due to interplay of systematic interactions, cell growth and division. Figure 4 shows that the nature of the trajectories are strikingly different when $p_{c}$ values are changed. For $p_{c}=10^{-5} \mathrm{~N} / \mathrm{m}$ in Figure $4 \mathrm{a}$, the cells move the smallest compared to $p_{c}=10^{-4} \mathrm{~N} / \mathrm{m}$ and $p_{c}=10^{-3} \mathrm{~N} / \mathrm{m}$ and are not persistent in nature. This is because the the memory effect in the $F A F$ is negligible, as illustrated in Figure 3 . The cells exhibit persistent trajectories for $p_{c}=10^{-4} \mathrm{~N} / \mathrm{m}$ 
and $p_{c}=10^{-3} \mathrm{~N} / \mathrm{m}$, with the degree of persistence being higher for the latter. The emergence of persistence in trajectories implies the presence of memory or (nonMarkovian) effects in the dynamics.

\section{CONCLUSION}

We have shown that in an evolving tissue colony, the dynamics of an individual cell may be approximated as a stochastic process where the force is correlated over many cell division times. The emergence of correlation in force is a manifestation of memory effects, which is a hallmark of non-Markovian dynamics. Memory effects in a growing tissue arises due to cell division and mechanical feedback. There is no potential or energy function whose derivative results in these processes. This immediately implies that there is no equilibrium in the growing tissue, and hence the system is always out of equilibrium. The present study also provides a mechanism for persistent motion observed in many out of equilibrium active matter systems 29$]$.

It is tempting to describe the simulated timedependent force autocorrelation using a reduced description, something like the Generalized Langevin equation (GLE), to describe the effective dynamics of a cell in the evolving tissue. It is natural to use such an approach in thermally controlled barrier crossing problems, as was done decades ago in the most insightful studies16117. For reasons stated above, construction of a similar set of equations, if it exists at all, in any reduced variable (the analogue of reaction coordinate in barrier crossing problems), which must also include large spatial heterogeneity, is likely to be difficult. Cell-division and apoptosis require energy input and dissipation, which can only be described using a physical picture and a framework that goes beyond the usual description based on Hamiltonians or energy functions. The work here may provide an impetus to develop a general theoretical framework for describing feedback controlled dynamics in active systems.

\section{ACKNOWLEDGEMENT}

We would like to thank Abdul N. Malmi-Kakkada and Himadri S. Samanta for valuable comments on the manuscript. This work was supported by grants from National Science Foundation (PHY 17-08128, PHY1522550). Additional support was provided by the CollieWelch Reagents Chair (F-0019).

\section{DATA AVAILABILITY}

The data that support the findings of this study are available from the corresponding author upon reasonable request.
${ }^{1}$ Ricard Alert and Xavier Trepat. Physical models of collective cell migration. Annual Review of Condensed Matter Physics, 11:77-101, 2020.

${ }^{2}$ M Reza Shaebani, Adam Wysocki, Roland G Winkler, Gerhard Gompper, and Heiko Rieger. Computational models for active matter. Nature Reviews Physics, 2(4):181-199, 2020.

${ }^{3} \mathrm{G}$ Hemlinger, PA Noetti, HC Lichtenbeld, RJ Melder, and RK Jain. Solid stress inhibits the growth of multicellular tumor spheroids. Nat. Biotech., 15:778-783, 1997.

${ }^{4}$ Morgan Delarue, Jörn Hartung, Carl Schreck, Pawel Gniewek, Lucy Hu, Stephan Herminghaus, and Oskar Hallatschek. Selfdriven jamming in growing microbial populations. Nature physics, 12(8):762-766, 2016.

${ }^{5}$ M. E. Dolega, M. Delarue, F. Ingremeau, J. Prost, A. Delon, and G. Cappello. Cell-like pressure sensors reveal increase of mechanical stress towards the core of multicellular spheroids under compression. Nature Communications, 8:14056 EP -, 012017.

${ }^{6}$ Alberto Puliafito, Lars Hufnagel, Pierre Neveu, Sebastian Streichan, Alex Sigal, D Kuchnir Fygenson, and Boris I Shraiman. Collective and single cell behavior in epithelial contact inhibition. Proceedings of the National Academy of Sciences, 109(3):739$744,2012$.

${ }^{7}$ Boris I Shraiman. Mechanical feedback as a possible regulator of tissue growth. Proceedings of the National Academy of Sciences, 102(9):3318-3323, 2005.

${ }^{8}$ Abdul N Malmi-Kakkada, Xin Li, Himadri S Samanta, Sumit Sinha, and Dave Thirumalai. Cell growth rate dictates the onset of glass to fluidlike transition and long time superdiffusion in an evolving cell colony. Physical Review X, 8(2):021025, 2018.

${ }^{9}$ Abdul Malmi-Kakkada, Xin Li, Sumit Sinha, and D Thirumalai. Dual role of cell-cell adhesion in tumor suppression and proliferation. arXiv:1906.11292, 2019.

${ }^{10}$ Sumit Sinha and D Thirumalai. Self-generated persistent random forces drive phase separation in growing tumors. The Journal of Chemical Physics, 153(20):201101, 2020.

${ }^{11}$ Sumit Sinha, Abdul N Malmi-Kakkada, Xin Li, Himadri S Samanta, and D Thirumalai. Spatially heterogeneous dynamics of cells in a growing tumor spheroid: Comparison between theory and experiments. Soft Matter, 16(22):5294-5304, 2020.

${ }^{12}$ Himadri S Samanta, Sumit Sinha, and D Thirumalai. Far from equilibrium dynamics of tracer particles embedded in a growing multicellular spheroid. arXiv:2003.12941, 2020.

${ }^{13}$ Sumit Sinha and Abdul N Malmi-Kakkada. Inter-particle adhesion regulates the surface roughness of growing dense threedimensional active particle aggregates. Journal of Physical Chemistry B, 125(37):10445-10451, 2021.

${ }^{14}$ Himadri S Samanta and D Thirumalai. Origin of superdiffusive behavior in a class of nonequilibrium systems. Physical Review E, 99(3):032401, 2019.

${ }^{15}$ Pawel Gniewek, Carl F Schreck, and Oskar Hallatschek. Biomechanical feedback strengthens jammed cellular packings. Physical review letters, 122(20):208102, 2019.

${ }^{16}$ John E Straub, Michal Borkovec, and Bruce J Berne. Calculation of dynamic friction on intramolecular degrees of freedom. Journal of Physical Chemistry, 91(19):4995-4998, 1987.

${ }^{17} \mathrm{JE}$ Straub, M Borkovec, and BJ Berne. Molecular dynamics study of an isomerizing diatomic in a lennard-jones fluid. $J$. Chem. Phys., 89:4833-4847, 1988.

${ }^{18}$ BJ Berne and GD Harp. On the calculation of time correlation functions. Advances in chemical physics, 17:63, 1970.

${ }^{19}$ DA Matoz-Fernandez, Kirsten Martens, Rastko Sknepnek, JL Barrat, and Silke Henkes. Cell division and death inhibit glassy behaviour of confluent tissues. Soft matter, 13(17):32053212, 2017.

${ }^{20}$ Dirk Drasdo and Stefan Höhme. A single-cell-based model of tumor growth in vitro: monolayers and spheroids. Physical biology, 2(3):133, 2005.

${ }^{21}$ Gernot Schaller and Michael Meyer-Hermann. Multicellular tumor spheroid in an off-lattice voronoi-delaunay cell model. Physical Review E, 71(5):051910, 2005. 
${ }^{22}$ Angela M Jimenez Valencia, Pei-Hsun Wu, Osman N Yogurtcu, Pranay Rao, Josh DiGiacomo, Inês Godet, Lijuan He, MengHorng Lee, Daniele Gilkes, Sean X Sun, et al. Collective cancer cell invasion induced by coordinated contractile stresses. Oncotarget, 6(41):43438, 2015.

${ }^{23}$ Rahul N Chacko, Peter Sollich, and Suzanne M Fielding. Slow coarsening in jammed athermal soft particle suspensions. Physical review letters, 123(10):108001, 2019.

${ }^{24}$ Xingbo Yang, M Lisa Manning, and M Cristina Marchetti. Aggregation and segregation of confined active particles. Soft matter, 10(34):6477-6484, 2014.

${ }^{25}$ James P Freyer and Robert M Sutherland. Regulation of growth saturation and development of necrosis in emt6/ro multicellular spheroids by the glucose and oxygen supply. Cancer research, 46(7):3504-3512, 1986.

${ }^{26}$ Joseph J Casciari, Stratis V Sotirchos, and Robert M Sutherland. Variations in tumor cell growth rates and metabolism with oxygen concentration, glucose concentration, and extracellular ph. Journal of cellular physiology, 151(2):386-394, 1992.

${ }^{27}$ Jacques Landry, James P Freyer, and Robert M Sutherland. Shedding of mitotic cells from the surface of multicell spheroids during growth. Journal of cellular physiology, 106(1):23-32, 1981.

${ }^{28}$ Jörg Galle, Markus Loeffler, and Dirk Drasdo. Modeling the effect of deregulated proliferation and apoptosis on the growth dynamics of epithelial cell populations in vitro. Biophysical journal, 88(1):62-75, 2005.

${ }^{29}$ Gerhard Gompper, Roland G Winkler, Thomas Speck, Alexandre Solon, Cesare Nardini, Fernando Peruani, Hartmut Löwen, Ramin Golestanian, U Benjamin Kaupp, Luis Alvarez, et al. The 2020 motile active matter roadmap. Journal of Physics: Condensed Matter, 32(19):193001, 2020.

${ }^{30}$ Clemens Bechinger, Roberto Di Leonardo, Hartmut Löwen, Charles Reichhardt, Giorgio Volpe, and Giovanni Volpe. Active particles in complex and crowded environments. Reviews of Modern Physics, 88(4):045006, 2016.

${ }^{31}$ M.C. Marchetti, J. F. Joanny, S. Ramaswamy, T.B. Liverpool, J. Prost, M. Rao, and R. Aditi Simha. Hydrodynamics of soft active matter. Rev. Mod. Phys., 85:1143, 2013. 

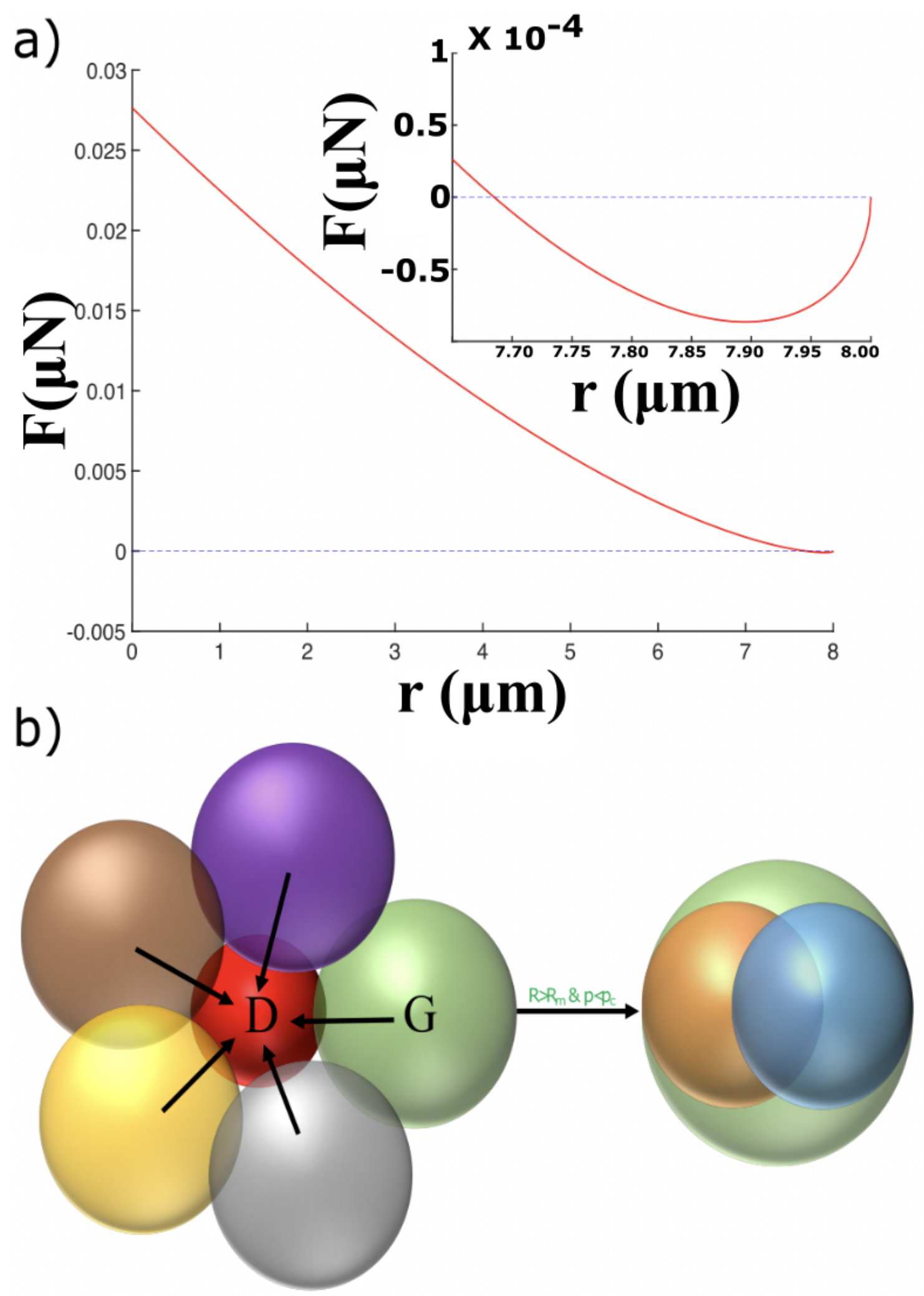

Figure 1: Schematic of the 2D model.(a) Total force (Eq. 33) as a function of inter-cellular distance for two cells, $i$ and $j$, with radius $R_{i}=R_{j}=4 \mu \mathrm{m}$. The repulsive and attractive part of the force are given by Eqs. 1 and 2 , respectively. The inset is the zoomed in view that highlights the region in which the force is predominantly attractive. (b) Cartoon illustrating the role of mechanical feedback. On the left, the 'red' cell is dormant (cannot grow and divide) because the pressure exerted by the neighbors exceeds $p_{c}$. The 'green' cell is in the growth phase $(G)$, which grows ands divides $\left(p<p_{c}\right)$. The green cell from the left gives given birth to two daughter cells (orange and cyan) when the radius exceeds the mitotic radius $R_{m}$. 


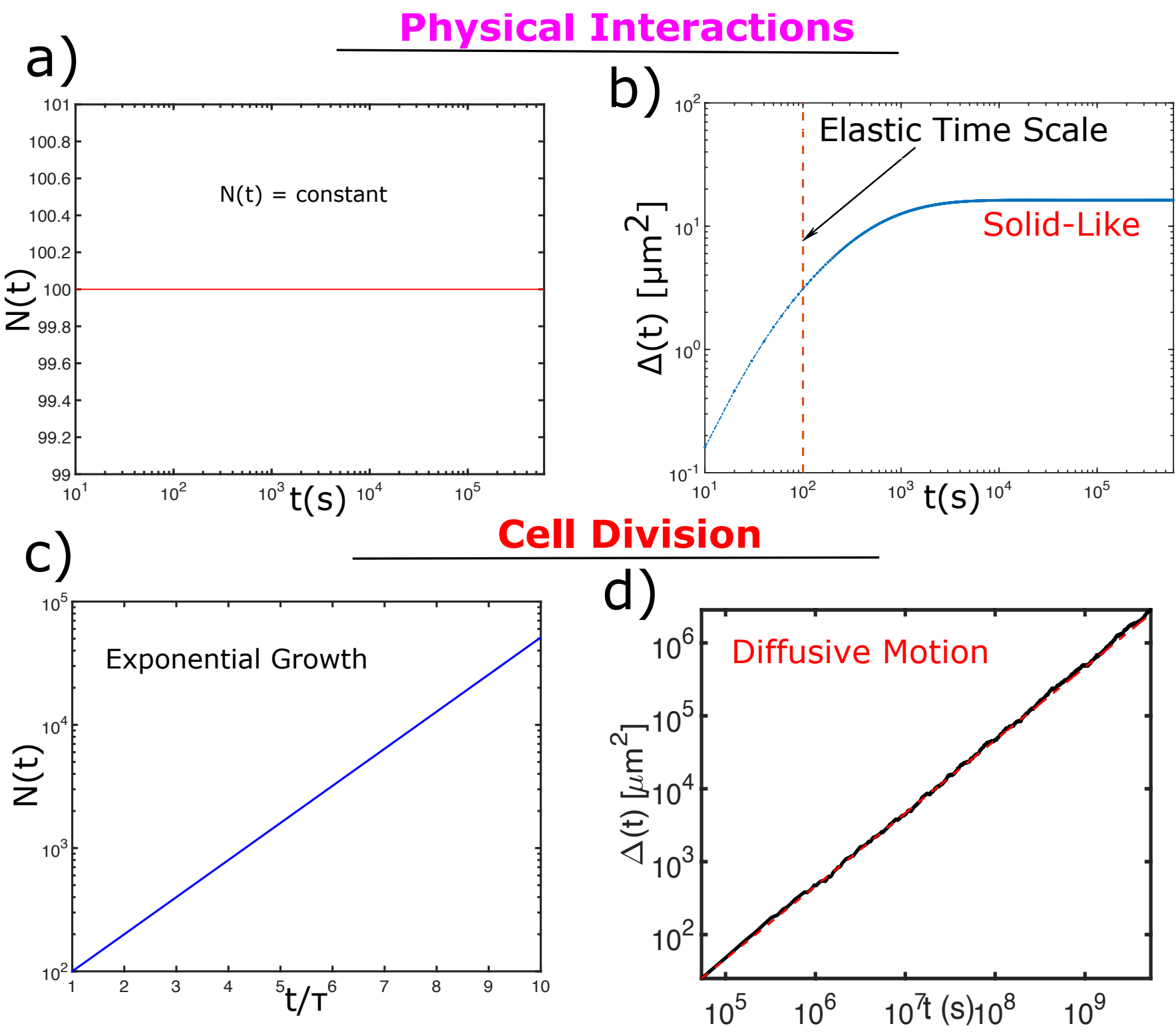

Figure 2: Number and MSD of cells solely based on physical interactions and cell division: (a) Number of cells, $N(t)$, solely based on physical two body interactions. Since, cell division rate is zero, $N(t)$ is a constant. (b) Mean-Squared Displacement, $\Delta(t)$, as a function of time when only physical interactions are present. $\Delta(t)$ relaxes initially, governed by elastic forces over a time-scale $\frac{\gamma}{E R_{m}}$ (red dashed vertical line), and settles to a plateau value. (c) $N(t)$ as a function of scaled time $\left(\frac{t}{\tau}\right)$ in the presence of cell division only. $N(t)$ grows exponentially. (d) $\Delta(t)$ as a function of time in the presence of cell division only. $\Delta(t)$ grows linearly in time and is diffusive (in black). The red dashed line is a linear fit given by Eq. 13 


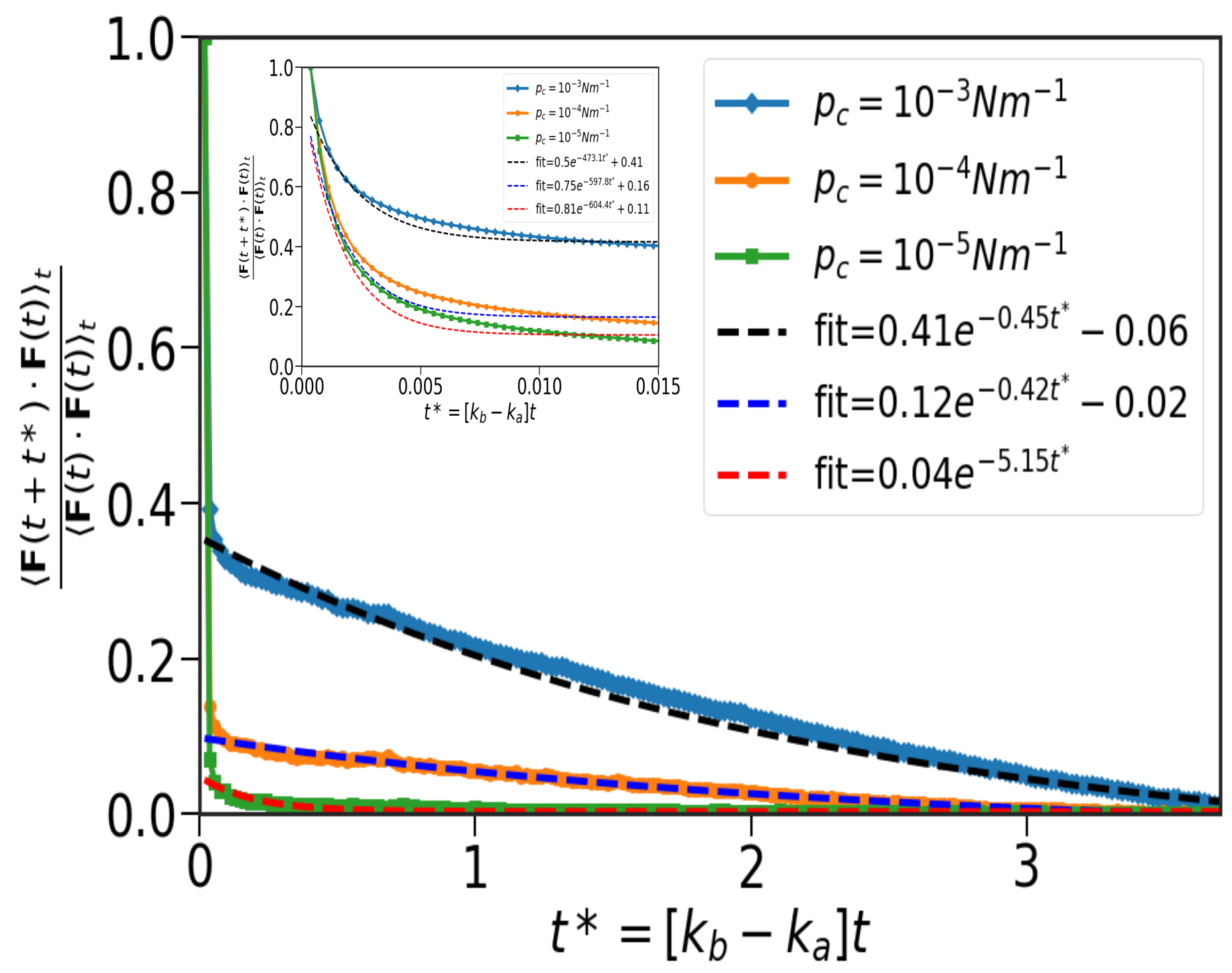

Figure 3: Emergence of highly correlated force: Plot of force auto-correlation function (FAF) as a function of time. From top to bottom, FAF corresponds to $p_{c}=10^{-3}, 10^{-4}$ and $10^{-5}$. The dashed line corresponds to the fits. In the inset, we zoom in on the initial time regime of FAF. The order of the plots and dashed lines are same as in the main figure. The figure shows the emergence of FAF with two time scales: one long $\left(\sim \frac{1}{k_{b}-k_{a}}\right)$ and one small (elastic time scale $\left.=\frac{\gamma}{E R_{m}}\right)$. 

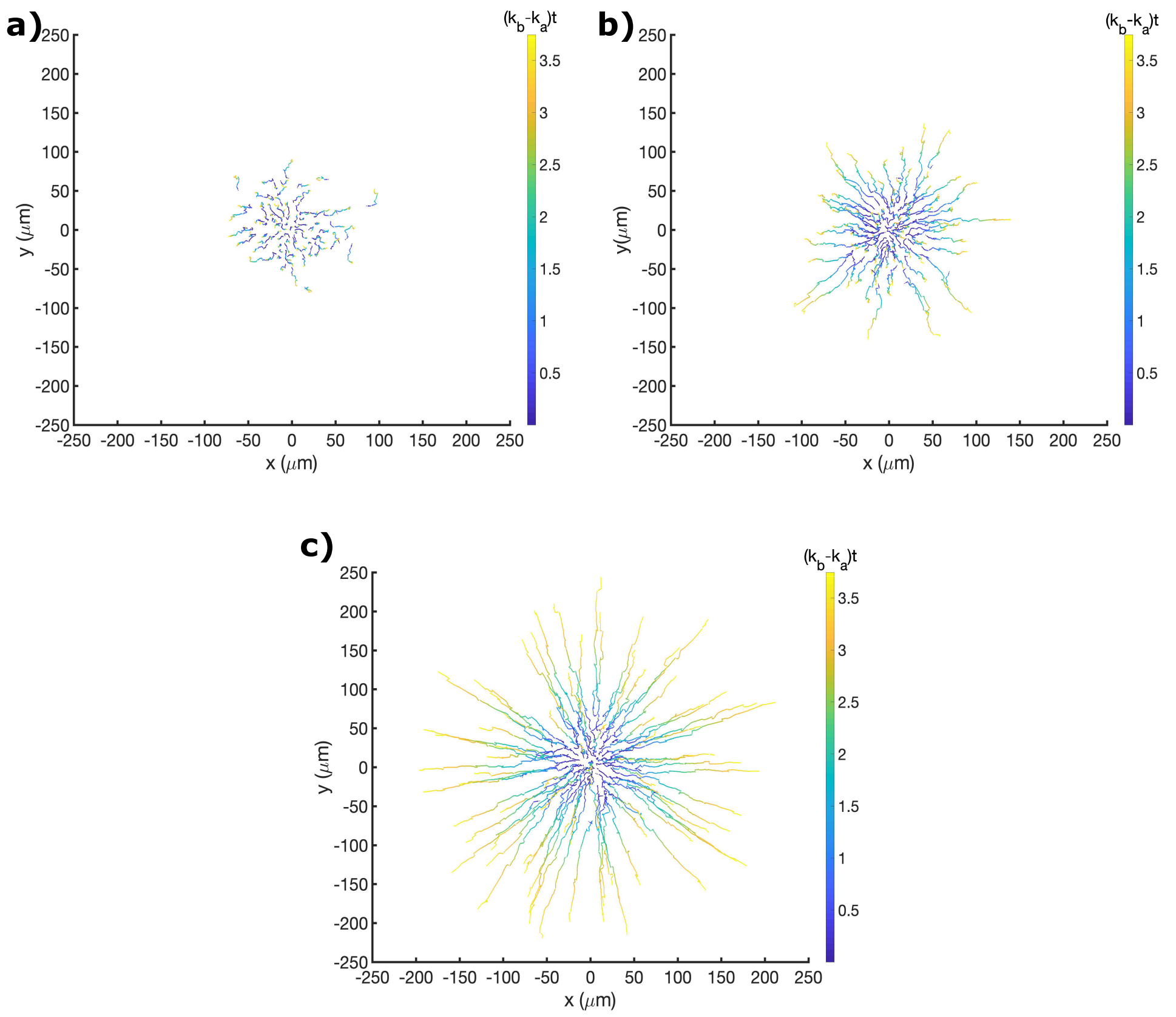

Figure 4: Trajectories of cells for different $p_{c}$ values: $(\mathbf{a}, \mathbf{b}, \mathbf{c})$ Trajectories of cells for $p_{c}=10^{-5} \mathrm{~N} / \mathrm{m}$, $p_{c}=10^{-4} \mathrm{~N} / \mathrm{m}$ and $p_{c}=10^{-3} \mathrm{~N} / \mathrm{m}$. For all the three panels, the color bar represents the time in units of $\left(k_{b}-k_{a}\right) t$. From the figures, it is clear that cells migration is enhanced when $p_{c}$ is increased. 